\title{
Simulation of disturbances and modelling of expected train passenger delays
}

\author{
A. Landex \& O. A. Nielsen \\ Centre for Traffic and Transport, \\ Technical University of Denmark, Denmark
}

\begin{abstract}
Forecasts of regularity for railway systems have traditionally - if at all - been computed for trains, not for passengers. It has only relatively recently become possible to model and evaluate the actual passenger delays. This paper describes how it is possible to use a passenger regularity model to estimate the actual passenger delays. The combination of the passenger regularity model with railway simulation software is described, demonstrating the possibility of predicting future passenger delays. The described passenger regularity model is run daily to calculate the passenger delays of the Copenhagen suburban rail network the previous day. The results obtained with the passenger regularity model used together with the simulation software are very similar to the daily calculated passenger regularity of the Copenhagen suburban network. As the combined method includes simulation software and reflects the actual passenger regularity, it is possible to use a combination of a passenger regularity model and simulation software to evaluate and compare future scenarios.
\end{abstract}

Keywords: railway planning, timetable, regularity, simulation, passenger delay.

\section{Introduction}

Relatively recently has it become possible to model and evaluate the actual passenger delay on large scale railway networks. The method used to model and evaluate actual passenger delays was presented in 2004 by Nielsen [2] and has since been optimised and evaluated $[3,4]$. In the planning process, the passenger delays are often calculated by assuming that no passengers transfer to other trains or update/change their route choice when delay or cancellations occur. This assumption does not reflect the passengers' travel behaviour. 
The first part of the paper briefly describes how to model passenger delays based on a comparison of realised timetables to planned timetables. A passenger strategy is presented, in which passengers plan their route according to the planned (announced) timetable, but start reconsidering their route within a certain threshold after a delay or cancellation of a train [4]. The route choice model, obtained from the passenger strategy, is run each night to evaluate passenger delays in the Copenhagen suburban rail network the previous day [5] and evaluates the impact of train delays on passengers. The model has shown that, due to delays caused by e.g. passenger/door interactions when timetables are stressed and when trains carries more passengers in the rush hours, passenger delays are greater than train delays [4].

Although the model presented in the first part of the paper is used to evaluate the already run timetable, the model can also be used for planning purposes. The second part of the paper describes how the model can be combined with railway simulation software such as RailSys, making it possible to predict the expected passenger delays for different timetable alternatives. The simulated timetables are exported to the passenger delay model for comparison with the planned timetable.

The last part of the paper demonstrates that a detailed timetable based passenger delay model together with railway simulation software can be used to evaluate different timetables in the planning process. The evaluation can estimate the expected train delays as well as the daily passenger delays. Furthermore, the model can be used to evaluate in which part of the network passenger delays pose problems.

\section{Calculating passenger delays}

The core idea of the model is modelling passenger delays by assigning a time-space trip matrix on the realised timetable. This is compared to a calculation where passengers were assigned on the planned timetable (the announced official timetable).

It is assumed that passengers plan their optimal desired route according to the planned timetable. If delays occur, exceeding a certain threshold, passengers are assumed to reconsider the route at that point in time and space along the route. If a train is completely cancelled, passengers reconsider their choice without a threshold.

As a benchmark (minimal passenger impact due to the delays), an optimal all-or-nothing route choice model can also be used on the realised timetable. This model assumes passengers to have full knowledge of future delays at the beginning of their trip and to choose optimally in accordance to this knowledge. The difference between the solutions obtained with the two methods (the optimistic and pessimistic) is a measurement of the additional loss of missing passenger information, combined with slow passenger responses to changes in the schedule. 


\section{Calculating passenger delays by simulation}

Calculating passenger delays of the actual performed operation is of interest to evaluate the train company and to identify aspects or routines that could be improved. If it is possible to predict or estimate the future passenger delays, it is possible to evaluate changes in the infrastructure and/or the timetables already when deciding new infrastructure and/or timetables. To evaluate infrastructure changes and timetables it is common to evaluate train delays by simulation. It would thus be obvious and interesting to calculate passenger delays in the same procedure.

To calculate the passenger delays by ordinary railway simulation software such as RailSys, it is necessary to build up the infrastructure and the timetables to be simulated. The rules of operation are then set up together with a set of delay distributions to simulate disturbances in the operation. It is now possible to run a simulation of the train operation with the chosen delay distributions. After running the simulation, it is possible to evaluate the infrastructure and the timetable whereupon improvements can be considered. The work process of the simulations can be seen in figure 1; the arrow describes the workflow.

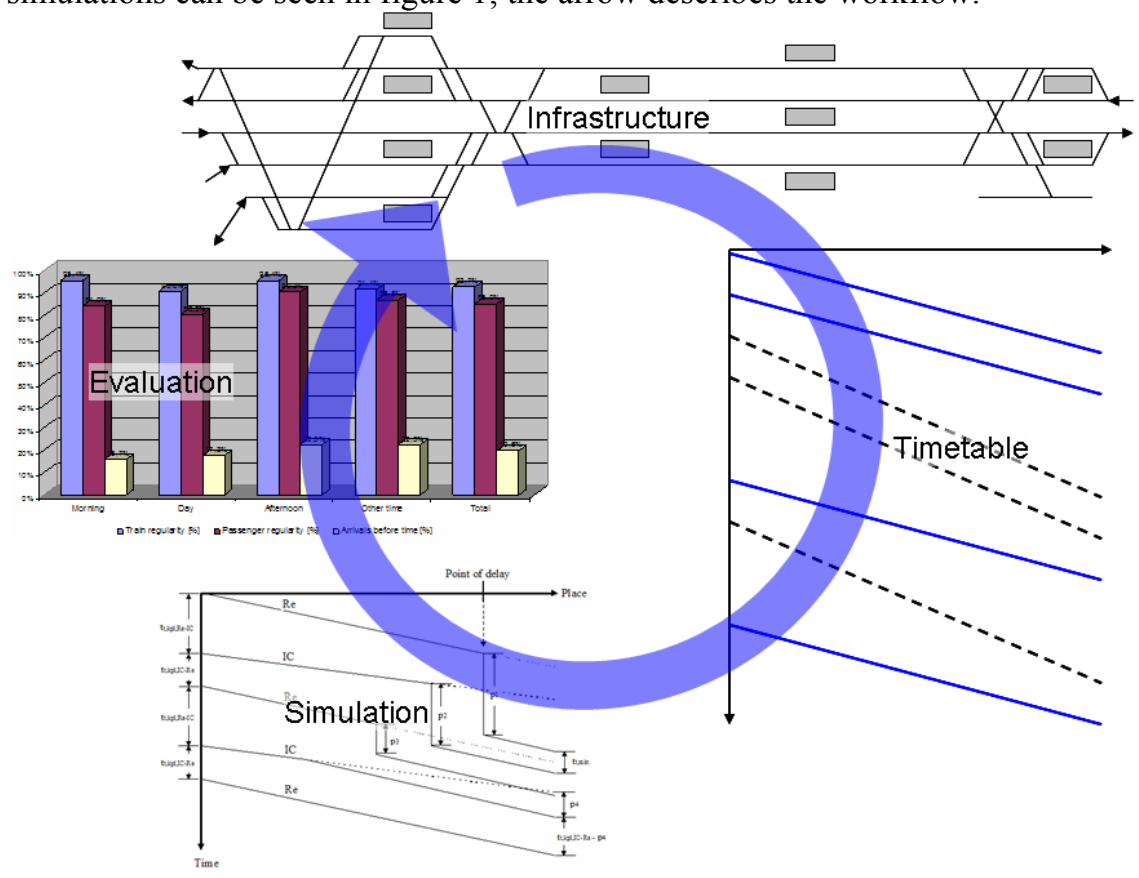

Figure 1: $\quad$ Principles of workflow in rail simulation projects.

Calculation of the passenger delays requires result data from the simulation to contain information of both the planned and all the realized/simulated timetables for all arrivals and departures. The RailSys output file Fahre++.pro contains this information. These results must be transferred from the railway simulation 
software to the passenger delay model by a simple import-export tool developed in VB.Net.

Calculation of passenger regularity is initiated by coding the infrastructure and creating the timetable. The rules of operation and the set of delay distributions are then defined. To ensure that the model reflect the real life operation, simulations are run and changes made in the rules of operations and the setup of delay distributions. When the model has been calibrated, the simulation is run. It is now possible to evaluate the train delays. However, to evaluate the passenger delays it is necessary to export the simulation data (the Fahre++.pro file in RailSys) to the passenger delay model before running the model. The workflow of calculating the passenger delays can be seen in figure 2 . The simulation of operation, export to passenger delay model and calculation of passenger delays simulates the impacts of one simulated day of operation. To calibrate the model and to obtain a delay distribution, it is therefore necessary to repeat the third step a number of times before the evaluation.

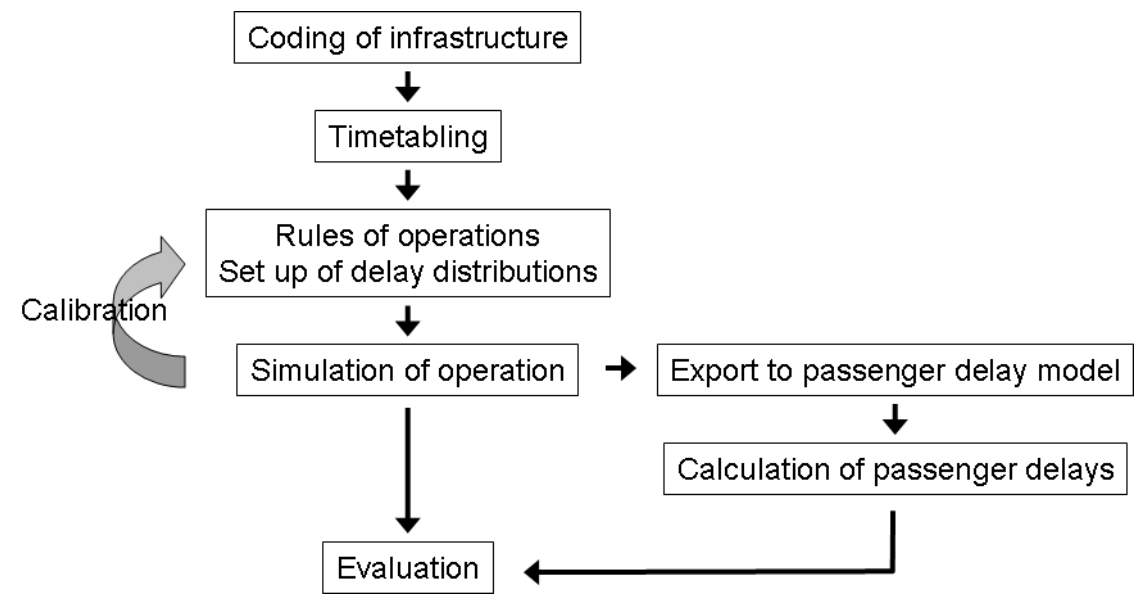

Figure 2: Workflow of simulating disturbances and modelling expected train passenger delays.

\section{Simulating disturbances on a large scale network}

The entire Copenhagen suburban rail network, including 85 passenger stations, was used for the simulations. The route network contains of 4 lines (A, B, C and E) running in 10 minutes service during the day (between 05:30 and 19:00 hours) and 20 minutes service during the rest of the day. 2 other lines $(\mathrm{G}$ and $\mathrm{H})$ run in 20 minutes service, and 1 line $(\mathrm{F})$ run in 5 minutes service in the daytime and 10 minutes service the rest of the day. Some of the departures on line $\mathrm{C}$ are shortened. The route network is seen in figure 3 . 


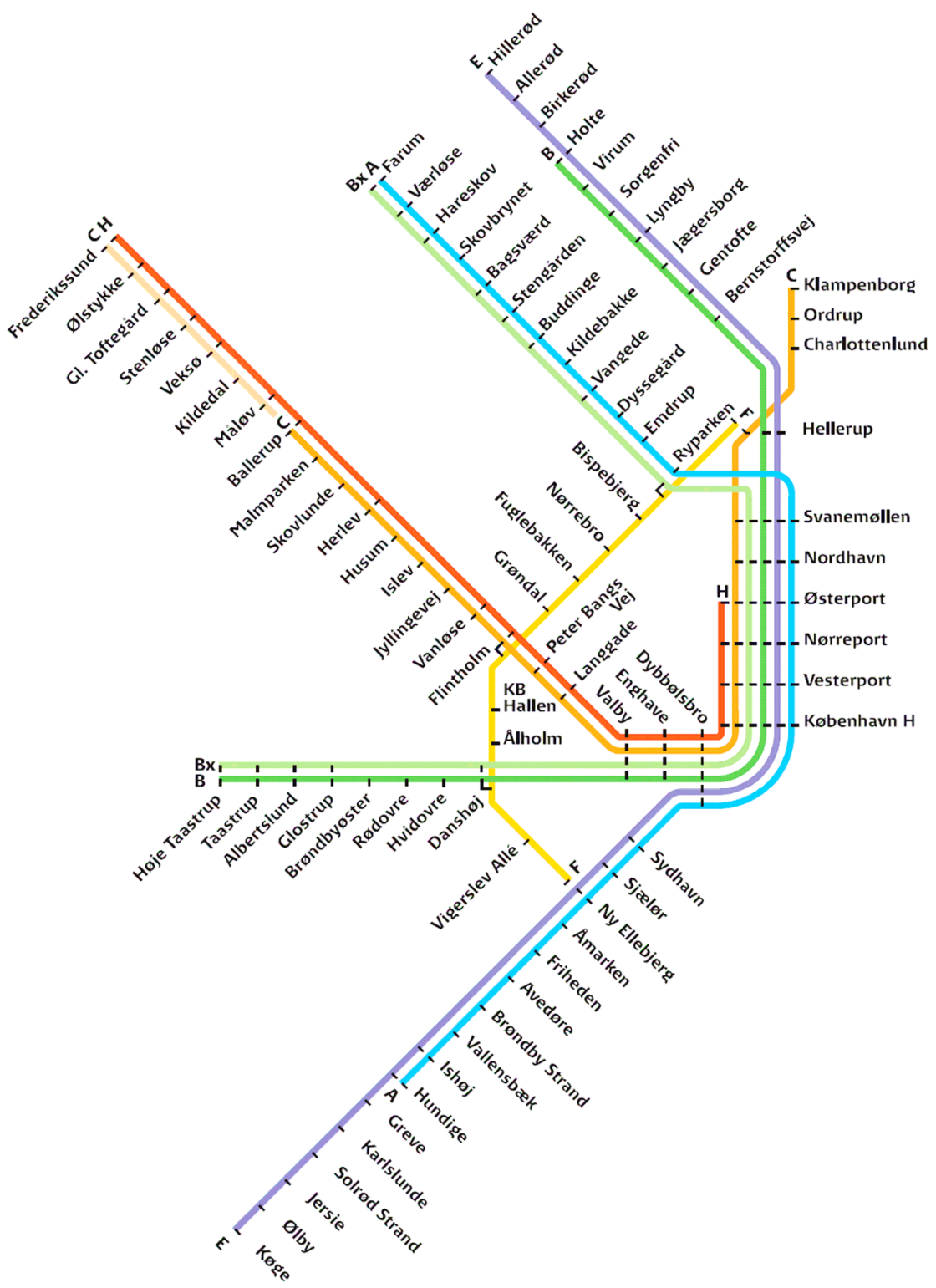

Figure 3: The Copenhagen suburban network, fall 2007.

\section{Results of simulating disturbances on large a scale network}

The RailSys model was run with 110 simulations, of which 2 contained deadlocks where trains blocked the way for each other. The remaining 108 simulations were used for further calculations and evaluations. 
The results show that the regularity of the trains is higher than the regularity of the passengers, cf. figure 4. The traditional way of calculating passenger regularity (multiplying the delay of the train and the expected getting off the train) is demonstrated to result in higher passenger regularity than when calculated by the passenger regularity model. The differences between the regularity between trains and passengers are due to different numbers of passengers in the trains through the day. Furthermore, some passengers have to change from one train to another under the risk of missing the other train.

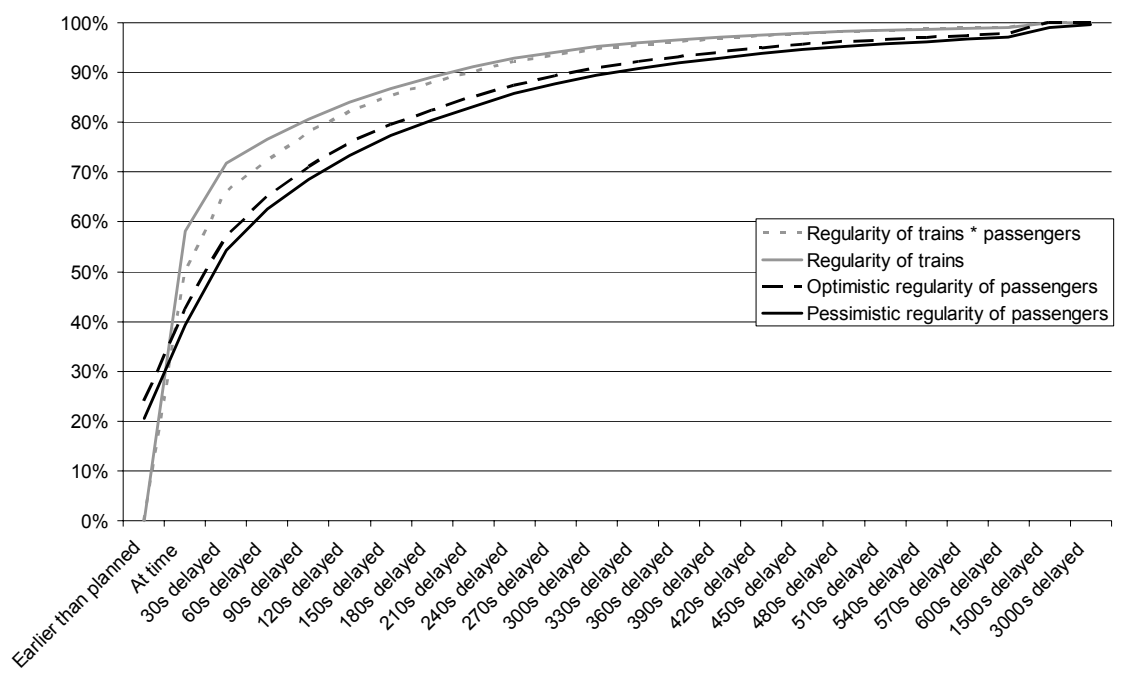

Figure 4: Regularity of trains and passengers at all stations.

Train delays do not necessarily cause passenger delays. Some passengers may even benefit from train delays. If a passenger arrives late to the station, a train delay may allow the passenger to catch an earlier train than expected. If the train catches up the delay, the passengers in the train may arrive on time. A similar situation may occur when a passenger changes from one line to another. If the train on the other line is delayed, it is possible to catch an earlier train than planned, thereby reducing the total travel time. In fact many passengers arrive earlier than planned (20 to $25 \%$ ), cf. figure 4 and figure 5 .

From figure 4 it is seen that the optimistic regularity of passengers in general is higher than the pessimistic regularity of passengers. The difference can be explained by the passengers' knowledge of the delays. In the optimistic calculations, full knowledge of the delays in the entire rail network is assumed to the extent that passengers have the information before the actual occurrence of the delays. In the pessimistic calculations passengers are assumed to follow a desired optimal route according to the timetable and only reconsider their route after a certain delay. Both principles of calculations have a certain error since passengers do not have full knowledge and passengers for some journeys choose the first train in their direction without waiting before reconsidering their route. 
Thus, the true regularity of passengers is between the optimistic and the pessimistic values.

The distribution of arrivals at stations according to the planned journey (cf. figure 5 (a)) once again shows that some passengers arrive before scheduled (negative delays). However, it is difficult to see a difference between the result of the optimistic and pessimistic calculation of the passenger delays. This difference is seen in figure $5 \mathrm{~b}$, illustrating a lesser tendency to delay and more passengers to arrive ahead of schedule when evaluated by the optimistic method rather than by the pessimistic method.
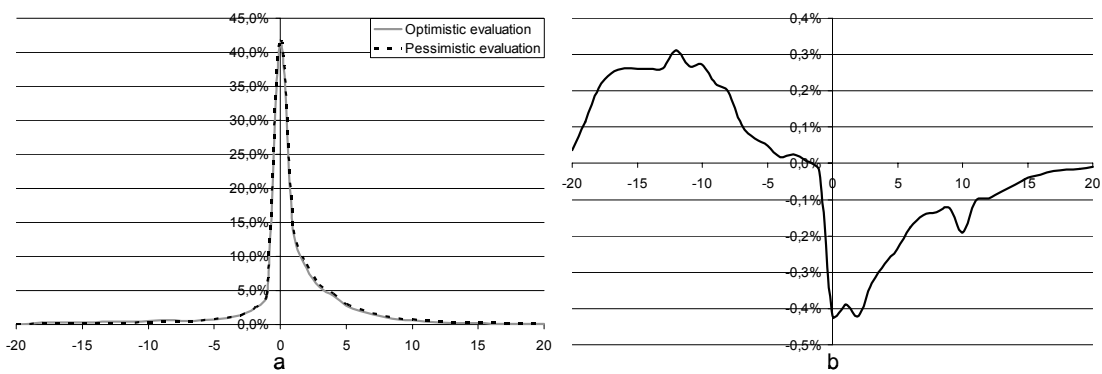

Figure 5: Distribution of arrivals according to the planned journey at all stations (a) and difference between optimistic and pessimistic evaluation of passenger regularity (b).

\section{Discussion}

Today, the passenger regularity model is run each night to evaluate passenger delays in the Copenhagen suburban network during the previous day [5]. The model has shown that passenger delays are larger than train delays [4], in accordance with the results presented in this paper. Other results (not published) show great similarity between the daily evaluation of delays and the simulated passenger delays.

Even though the RailSys model reproduces the results in Copenhagen quite well, the results can be improved. To do this and to improve reproducibility of the results, the RailSys model must be further calibrated to make the resulting delay at all stations similar to the daily operation. The RailSys model used in this paper has only been calibrated on an overall level so that the average delay for all stations is equal to the daily operation. It is very time-consuming, approaching the impossible, to gain exactly the same delay distribution as for the daily operation and the calibration should thus "only" be at the same level as (and not exact) the regularity of the daily operation [1].

When the RailSys model is calibrated, it is possible to evaluate the regularity of both trains and passengers at isolated stations as shown in figure 6. Beyond that, the passenger regularity model can be used for evaluating (and ranking) infrastructure improvements. The benefits for the passengers in terms of travel time and delays can be estimated and compared with the construction costs in, 
e.g., a cost-benefit analysis. Furthermore, different candidate timetables can be evaluated and compared in the process of developing the best possible timetable for the passengers.

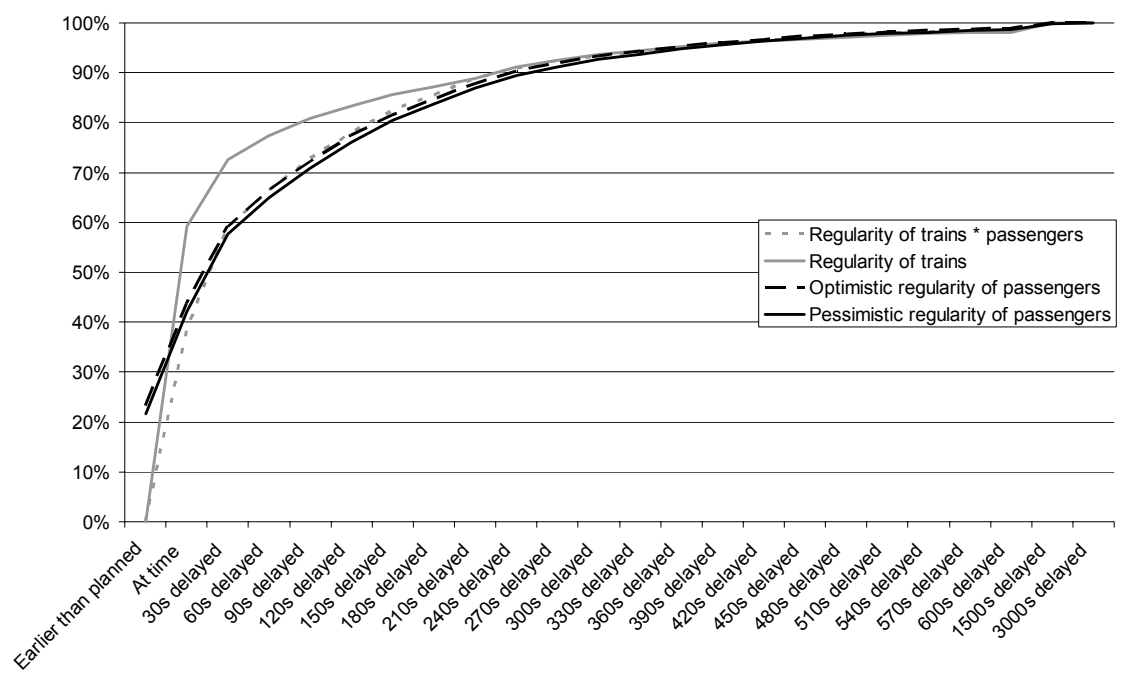

Figure 6: Regularity of trains and passengers at Copenhagen central station (København H).

Apart from the RailSys model, the passenger regularity model itself can be improved. According to the passenger regularity model, passengers will not change their route of travelling until a certain threshold of delay has been reached. However, on some stations or OD-relations, passengers will just take the first train in their direction. This phenomenon is characteristic for short journeys with high train frequency and is observed in the central Copenhagen between Østerport and Vesterport (cf. figure 3) with a train frequency of 2 minutes in each direction. The phenomenon might, however, also be observed at OD-relations with a lower frequency - i.e. Lyngby-Nørreport (cf. figure 3). Further work is necessary to estimate the correct threshold of delay to make passengers reconsider their route.

\section{Conclusions}

We have shown that it is possible to calculate the expected passenger delays by simulation of large scale networks and that there is a significant difference between train regularity and passenger delays.

The difference between the train regularity and passenger delays is due to the different number of passengers in the trains during the day and the fact that the passengers (to some extent) will change routes due to delays. Furthermore, there is a higher risk of delays in the rush hours due to more passengers and more trains. 
The evaluation of passenger obtained with a simulation software, RailSys, and the passenger regularity model is comparable to the daily operation of the Copenhagen suburban network. Using a well calibrated RailSys model it will be possible to compare travel times and delays for different future scenarios - for changes in infrastructure as well as in timetables. In this way it will be possible to choose the best possible scenario.

Even though the results in this paper are very similar to what has been observed on the Copenhagen suburban rail network, the results can be improved both by better calibration of the RailSys model and estimation of the correct threshold of delay before reconsidering the route.

\section{Acknowledgements}

Rapidis Aps is thanked for the programming the passenger delay model. Rail Net Denmark (Banedanmark) is thanked for providing the infrastructure data for the Copenhagen urban rail network and discussions on the RailSys model. DSB Stog (the train company of the Copenhagen suburban rail network) is thanked for providing the future timetables and OD-matrices for the travel patterns. Stephen Hansen and Kenneth Christensen, Centre for Traffic and Transport at the Technical University of Denmark, is thanked for developing the import-export tool from RailSys to the passenger delay model and the evaluation tool used.

\section{References}

[1] Kaas, A. H., Punctuality model for railways. Proc. of the $7^{\text {th }}$ International Conference on Computers in Railways, eds. J. Allan, R. J. Hill, C. A. Brebbia, G. Sciutto \& S. Sone, pp. 853-860, 2000

[2] Nielsen, O. A., A large scale stochastic multi-class schedule-based transit model with random coefficients. Schedule-Based Dynamic Transit Modelling - Theory and Applications. In Schedule-Based Dynamic Transit Modelling: theory and applications, eds. Wilson, N. and Nuzzolo, A. Kluwer Academic. pp. 51-77, 2004

[3] Nielsen, O. A. \& Frederiksen, R. D., Optimisation of timetable-based, stochastic transit assignment models based on MSA. Paper accepted for Annals of Operations Research special issue on Optimisation in Transportation. Forthcoming, Elsevier, 2006

[4] Nielsen, O. A. \& Frederiksen, R. D., Modelling train passenger delays. Symposium on The Reliability of Travelling and the Robustness of Transport Systems, eds. van Zuylen, H.J., pp. 137-156, 2005

[5] Seest, E., Nielsen, O. A. \& Frederiksen, R. D., Calculating passenger regularity in the Copenhagen suburban network. Proc. of Trafficdays, 2005 (in Danish). 